\title{
Pulmonale Hypertonie und schlafbezogene Atmungsstörungen
}

\author{
R. Schulz \\ H. J. Eisele \\ N. Weissmann \\ W. Seeger
}

Pulmonary Hypertension and Sleep-Related Breathing Disorders

\section{Zusammenfassung}

Eine pulmonale Hypertonie, d.h. eine Erhöhung des mittleren Pulmonalarteriendruckes in Ruhe über einen Wert von $20 \mathrm{~mm} \mathrm{Hg}$, kann bei verschiedenen Formen schlafbezogener Atmungsstörungen beobachtet werden. Bei der obstruktiven Schlaf-Apnoe (OSA) bewirken die Apnoe-assoziierten Trigger von Hypoxie und intrathorakalen Druckschwankungen repetitive Anstiege der pulmonalarteriellen Drucke im Schlaf. Bei 20 - 30\% der OSA-Patienten ist auch am Tage eine pulmonalarterielle Hypertonie vorhanden. Diese ist meistens nur gering ausgeprägt und führt nur selten zum klinisch manifesten Cor pulmonale. Eine effektive CPAP-Therapie besitzt einen günstigen Einfluss auf die pulmonale Hypertonie bei OSA. Bei der schweren Linksherzinsuffizienz (LVEF $<40 \%$ ) kann es im Rahmen einer pulmonalvenösen Hypertonie zur Stimulation von pulmonalen „stretch“ und „irritant"-Rezeptoren kommen. Die nachfolgende Hyperventilation führt zum Absinken der $\mathrm{pCO}_{2}$-Werte unter die Apnoe-Schwelle und damit zur Entstehung einer Cheyne-Stokes-Atmung (CSR) bei bis zur Hälfte dieser Patienten. Bei Patienten mit fortgeschrittener idiopathischer pulmonalarterieller Hypertonie (IPAH) kann ein der CSR ähnliches pathologisches Atemmuster im Schlaf beobachtet werden. Pathogenetische Faktoren dieser periodischen Atmung sind wahrscheinlich ebenfalls verlängerte Zirkulationszeiten und Hypokapnie. Zusammenfassend können schlafbezogene Atmungsstörungen einerseits Ursache einer pulmonalen Hypertonie sein (OSA-assoziierte pulmonale Hypertonie), andererseits kann eine pulmonale Hypertonie jedoch auch selbst zur Entstehung schlafbezogener Atmungsstörungen führen (CSR bei Linksherzinsuffizienz, periodische Atmung bei IPAH).

\section{Abstract}

Pulmonary hypertension ( $\mathrm{PH}$ ), i.e. an increase of mean pulmonary artery pressure above $20 \mathrm{~mm} \mathrm{Hg}$ under resting conditions, can be observed in different forms of sleep-disordered breathing (SDB). In obstructive sleep apnea (OSA) the apnea-associated triggers of hypoxia and intrathoracic pressure swings lead to repetitive rises of pulmonary artery pressure during sleep. In $20-30 \%$ of these patients daytime PH occurs. PH in the setting of OSA is usually mild and rarely causes clinically evident cor pulmonale. Effective CPAP therapy has a beneficial influence on pulmonary hemodynamics in OSA. Severe congestive heart failure (i.e. with a $\operatorname{LEEF}<40 \%$ ) might provoke pulmonary venous hypertension and thereby stimulation of pulmonary stretch and irritant receptors. The ensuing hyperventilation leads to a decrease of $\mathrm{pCO}_{2}$ levels below the apneic threshold and thus contributes to the emergence of Cheyne Stokes respiration (CSR) in up to one half of the affected patients. Patients suffering from advanced idiopathic pulmonary arterial hypertension (IPAH) might show a similar breathing pattern while asleep. Possible pathogenetic factors of the nocturnal periodic breathing occurring in end-stage IPAH are prolonged circulation times and hypocapnia. In conclusion, SDB might cause PH (OSA-associated PH). On the other hand, PH might lead to the development of SDB (CSR in congestive heart failure, periodic breathing in IPAH). 


\section{Einleitung}

In der aktuellen medizinischen Literatur wird unter dem Thema „pulmonale Hypertonie und schlafbezogene Atmungsstörungen“ im Allgemeinen die Entwicklung einer pulmonalarteriellen Hypertonie im Rahmen der obstruktiven Schlaf-Apnoe (OSA) verstanden. So widmet sich ein kürzlich veröffentlichter GuidelineBericht des American College of Chest Physicians fast ausschließlich diesem Aspekt der Interaktion zwischen Lungengefäßen und gestörter Atmung im Schlaf [1]. Wir möchten in dem vorliegenden Essay aufzeigen, dass es noch andere Zusammenhänge zwischen pulmonaler Hypertonie und schlafbezogenen Atmungsstörungen gibt, die bisher noch wenig beachtet und untersucht worden sind. Hierbei handelt es sich um die Entstehung der Cheyne-Stokes-Atmung (CSR) im Rahmen einer pulmonalvenösen Hypertonie bei schwerer Linksherzinsuffizienz und das Phänomen der nächtlichen periodischen Atmung bei Patienten mit idiopathischer pulmonalarterieller Hypertonie (IPAH; früher: primäre pulmonale Hypertonie). Auf den Themenkomplex der pulmonalen Hypertonie bei OSA möchten wir hier nur summarisch eingehen, da er an anderer Stelle schon ausführlicher behandelt wurde $[2,3]$.

\section{Obstruktive Schlaf-Apnoe und pulmonale Hypertonie}

Die OSA bewirkt während der Nacht repetitive Anstiege des pulmonalarteriellen (PA-)Druckes, die während der post-apnoischen Hyperventilationsphasen ihr Maximum erreichen. Als wesentliche Trigger dieser hämodynamischen Veränderungen werden Hypoxie und intrathorakale Druckschwankungen angesehen $[4,5]$.

Darüber hinaus kann die OSA auch eine am Tage anhaltende pulmonale Hypertonie hervorrufen ([6-16], s. Tab.1). Die ersten Kollektive, die bezüglich dieser Fragestellung untersucht wurden, wiesen eine PA-Druckerhöhung bei 20-30\% der OSA-Pa- tienten nach. Es handelte sich hierbei vor allem um Patienten mit gleichzeitig vorhandener Ventilationsstörung (COPD, obesity hypoventilation bzw. Pickwick-Syndrom) und konsekutiver Veränderung der Blutgase im Sinne einer respiratorischen Partialoder Globalinsuffizienz [7-10,13]. Deshalb wurden Zweifel geäußert, ob die OSA per se eine pulmonale Hypertonie am Tage verursachen kann. Mittlerweile sind jedoch Studien durchgeführt worden, die nur Patienten mit normaler Lungenfunktion einschlossen und ähnliche Prävalenzraten der pulmonalen Hypertonie bei OSA nachweisen konnten [11,12,14-16].

Ein weiteres Argument für einen ursächlichen Zusammenhang zwischen dem Vorhandensein einer OSA und der Entwicklung einer pulmonalen Hypertonie ist, dass die PA-Drucke unter einer effektiven CPAP-Therapie abnehmen [15,17]. Schließlich wurde tierexperimentell sowohl bei der Ratte als auch bei der Maus gezeigt, dass der OSA-assoziierte Stimulus einer chronisch-intermittierenden Hypoxie eine am Tage persistierende pulmonale Hypertonie auslösen kann $[18,19]$.

Der Schweregrad der pulmonalen Hypertonie bei OSA ist in der Regel nur gering; die zitierten Studien beschreiben mittlere PADrucke, die nicht höher als $30 \mathrm{~mm} \mathrm{Hg}$ liegen. Folglich leiden im Gegensatz zur teilweise immer noch verbreiteten Lehrmeinung auch nur wenige OSA-Patienten an einem klinisch manifesten Cor pulmonale.

Eine Korrelation zwischen OSA-Schweregrad (ausgedrückt als Apnoe-Hypopnoe-Index [AHI] oder Ausmaß der nächtlichen Entsättigungen) und PA-Druck ist bisher nicht gefunden worden.

Dies könnte dadurch bedingt sein, dass die genannten polysomnographischen Parameter das Ausmaß der OSA nur unzureichend widerspiegeln und/oder von Nacht zu Nacht signifikanten Schwankungen unterworfen sein können. Ein weiterer Grund für die fehlende Korrelation zwischen OSA-Schweregrad und PADruck könnte sein, dass das Ausmaß der OSA-assoziierten pul-

Tab. 1 Studien zur Prävalenz einer am Tage vorhandenen pulmonalen Hypertonie bei obstruktiver Schlaf-Apnoe

\begin{tabular}{|c|c|c|c|c|c|}
\hline $\begin{array}{l}\text { Autor/Jahr } \\
\text { Podszus/1986 }\end{array}$ & \multicolumn{2}{|c|}{$\begin{array}{l}\text { Prävalenz PH } \\
\text { (Anzahl der Patienten mit PH/Gesamtzahl } \\
\text { der untersuchten Patienten [\%]) }\end{array}$} & $\begin{array}{l}\text { mittlerer PA-Druck } \\
\text { (mmHg) } \\
29\end{array}$ & $\begin{array}{l}\text { Methodik zur } \\
\text { Erfassung der PH }\end{array}$ & $\begin{array}{l}\text { Einschluss von } \\
\text { Patienten mit } \\
\text { COPD/OHS } \\
?\end{array}$ \\
\hline Weitzenblum/1988 & $9 / 46$ & [20] & 23 & Katheter & ja \\
\hline Krieger/1989 & 19/100 & [19] & 30 & Katheter & ja \\
\hline Sanner/1997 & $18 / 92$ & [20] & 22 & Katheter & nein \\
\hline Sajkov/1999 & $11 / 32$ & [34] & 24 & Doppler & nein \\
\hline Niijima/1999 & $10 / 19$ & [53] & 29 & Katheter & ja \\
\hline Bady/2000 & $12 / 44$ & [27] & 29 & Katheter & nein \\
\hline Alchanatis/2001 & $6 / 29$ & [21] & 26 & Doppler & nein \\
\hline Yamakawa/2002 & $8 / 37$ & [22] & $?$ & Doppler & nein \\
\hline
\end{tabular}

$\mathrm{PH}$ = pulmonale Hypertonie, PA-Druck = pulmonalarterieller Druck, COPD = chronisch-obstruktive Lungenerkrankung, OHS = obesity hypoventilation-Syndrom 


\section{linksventrikuläre Dysfunktion

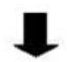 \\ pulmonalvenöse Rückstauung}

Stimulation von pulmonalen stretch - und irritant - Rezeptoren
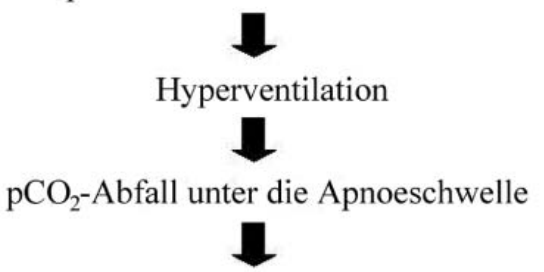

Cheyne-Stokes-Atmung

Abb. 1 Hypothese zur Entstehung der Cheyne-Stokes-Atmung im Rahmen einer pulmonalvenösen Hypertonie.

monalen Hypertonie von der individuellen Stärke der hypoxischen pulmonalen Vasokonstriktion beeinflusst wird. Dies legt eine Studie nahe, bei der die PA-Drucke bei OSA-Patienten echokardiographisch nach Inspiration von Gasgemischen mit unterschiedlichen $\mathrm{O}_{2}$-Konzentrationen gemessen wurden [12].

\section{Cheyne-Stokes-Atmung und pulmonale Hypertonie}

Die CSR wird zu den schlafbezogenen Atmungsstörungen ohne Obstruktion der oberen Atemwege gezählt. Sie wird bei $40-50 \%$ der Patienten mit chronischer Herzinsuffizienz und einer linksventrikulären Ejektionsfraktion $<40 \%$ beobachtet $[20,21]$. Die Pathophysiologie der CSR ist bis heute im Detail noch nicht verstanden, wesentliche Rollen werden allerdings verlängerten Zirkulationszeiten und einer Hypokapnie zugeschrieben. Eine Verlängerung der Zirkulationszeiten im Rahmen der eingeschränkten linksventrikulären Funktion führt zu einer verzögerten Weitergabe von chemischen Atemstimuli an die entsprechenden Rezeptoren, wodurch das Feedback-System der Atmungssteuerung in Oszillationen versetzt wird [22]. Dies findet ihren Ausdruck in den typischen Crescendo-Decrescendo-Hyperventilationsphasen der CSR. Für das Auftreten der Apnoen ist nach heutiger Meinung hingegen eine Hypokapnie verantwortlich [23,24]. Dabei wird folgende pathophysiologische Sequenz angenommen (s. Abb. 1).

Im Rahmen der Funktionsstörung des linken Ventrikels kommt es durch Rückstau von Blut in das pulmonale Gefäßbett zur Stimulation von sog. „stretch“- und „irritant“-Rezeptoren. Dies bewirkt eine Hyperventilation mit Absinken des $\mathrm{pCO}_{2}$-Wertes unter die Apnoeschwelle und damit das Auftreten zentraler Apnoen.

Diese Hypothese wird durch mehrere Arbeiten gestützt. So konnte von unterschiedlichen Arbeitsgruppen gezeigt werden, dass Herzinsuffizienz-Patienten mit CSR höhere pulmonalkapilläre Verschlussdrucke (PCWP) als solche ohne CSR aufweisen [25 - 29]. Zudem wurde eine lineare Korrelation des PCWP mit dem AHI festgestellt [30]. Weiterhin wurde gefunden, dass die $\mathrm{pCO}_{2}$-Werte bei diesen Patienten umso niedriger sind, je höher der PCWP ist [30]. Schließlich konnte in einer echokardiographischen Studie demonstriert werden, dass CSR-Patienten vergrößerte rechtsventrikuläre Dimensionen und eine reduzierte RVFunktion im Vergleich zu Herzinsuffizienz-Patienten ohne nächtliche Atmungsstörung haben [31]. Auch dies sind Hinweise für die Bedeutung einer pulmonalvenösen Hypertonie in der Pathogenese der CSR.

Idiopathische pulmonalarterielle Hypertonie und nächtliche periodische Atmung

Die idiopathische pulmonalarterielle Hypertonie (IPAH) ist eine seltene Erkrankung ungeklärter Ätiologie, bei der es zu einer Widerstandserhöhung präkapillärer pulmonaler Arteriolen mit der Folge einer Druckbelastung des rechten Herzens kommt [32]. Die IPAH betrifft überwiegend jüngere Frauen und führt unbehandelt innerhalb weniger Monate bzw. Jahre zum progredienten Rechtsherzversagen.

Durch eine Reduktion der rechtsventrikulären Ejektionsfraktion sowie des Herzzeitvolumens führt die IPAH zur Verlängerung der Zirkulationszeiten. Weiterhin findet man blutgasanalytisch oft eine Hypoxämie und eine Hypokapnie. Ursache der Hypoxämie ist in erster Linie eine Reduktion der Gasaustauschfläche, während die Hypokapnie durch eine kompensatorische Hyperventilation hervorgerufen wird. Insofern liegen also bei der IPAH ähnliche Faktoren vor, wie sie für die Entstehung der CSR bei Linksherzinsuffizienz verantwortlich gemacht werden. Bisher war die nächtliche Atmung jedoch bei IPAH-Patienten noch nicht polysomnographisch untersucht worden. Bekannt war lediglich, dass diese Patienten im Schlaf Desaturationen aufweisen können [33].

Wir haben in einer Pilotstudie 20 konsekutive IPAH-Patienten, die zu einer Rechtsherzkatheter-Untersuchung mit pharmakologischer Testung aufgenommen worden waren, polysomnographisch untersucht [34]. Sechs der 20 Patienten (30\%) zeigten eine nächtliche periodische Atmung, wobei hiervon diejenigen Patienten mit schwerer IPAH betroffen waren. Patienten mit periodischer Atmung hatten höhere mittlere PA-Drucke, höhere pulmonalvaskuläre Widerstände und niedrigere Herzzeitvolumina. In Abb. 2 ist die polysomnographische Registrierung bei einer Patientin mit IPAH und periodischer Atmung beispielhaft dargestellt.

Bei 4 der 6 Patienten konnte die periodische Atmung durch $\mathrm{O}_{2}$-Gabe via Nasensonde fast vollständig zur Rückbildung gebracht werden. Bei einer Patientin mit periodischer Atmung wurde eine Normalisierung des Atemmusters nach erfolgreicher Lungentransplantation festgestellt [35]. Diese kasuistischen Beobachtungen sind mit Daten zur CSR bei Linksherzinsuffizienz vergleichbar, die durch $\mathrm{O}_{2}$-Gabe oder nach Herztransplantation reversibel sein kann [36-38]. Der therapeutische Effekt der $\mathrm{O}_{2}$-Gabe kommt hierbei vermutlich durch eine Hemmung des hypoxischen Atemantiebes mit konsekutiver Anhebung der $\mathrm{pCO}_{2}$-Werte über die Apnoeschwelle zustande.

Zukünftige Untersuchungen an größeren Patientenkollektiven müssen klären, wie häufig die periodische Atmung bei IPAH ist 


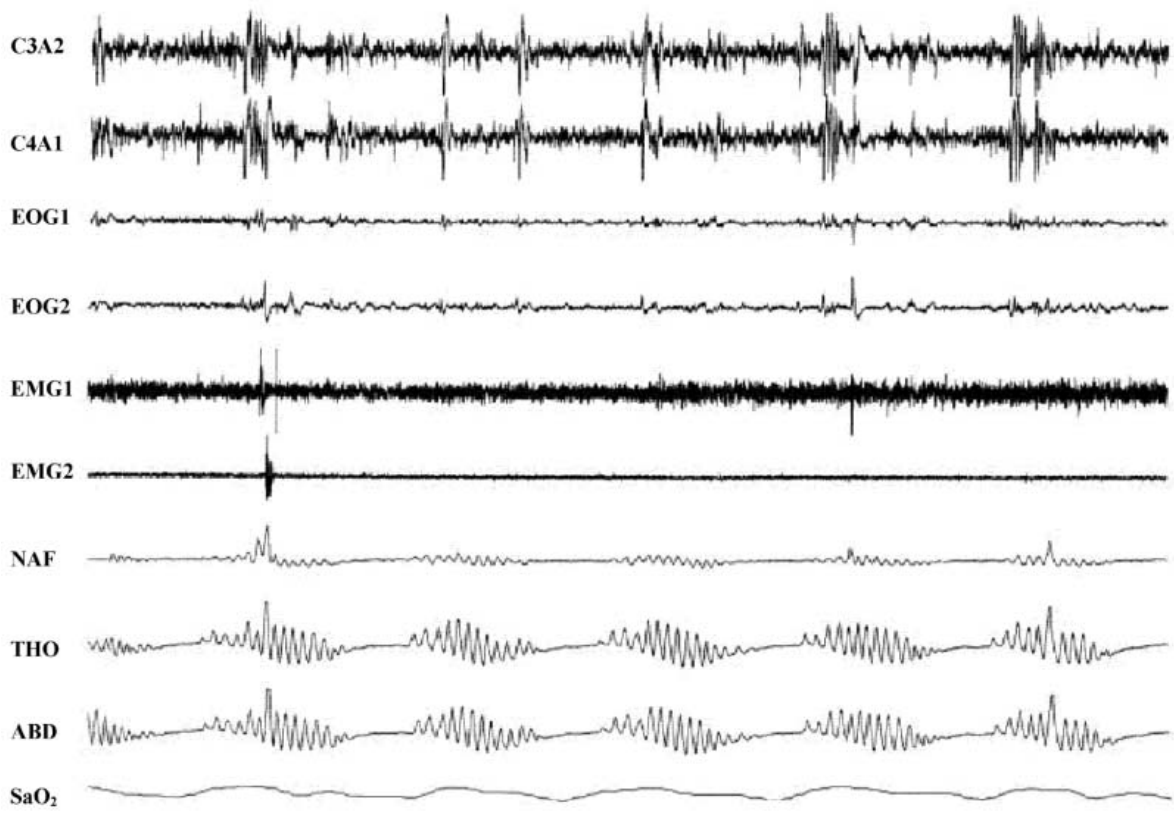

Abb. 2 Nächtliche periodische Atmung bei einer Patientin mit idiopathischer pulmonaler Hypertonie (Polysomnographische Registrierung, 5 min.-Ausschnitt; C3A2/C4A $1=$ EEG-Kanäle, EOG1/2 = Elektrookulogramm, EMG1/2 = submentales und Bein-Elektromyogramm, NAF = oronasaler Atemfluss, $\mathrm{THO} / \mathrm{ABD}=$ Thorax- und Abdomenexkursionen, $\mathrm{SaO}_{2}=\mathrm{O}_{2}$-Sättigung).

und welche klinische und prognostische Bedeutung ihr $\mathrm{zu}$ kommt. So könnte u. a. vermutet werden, dass die pulmonale Hämodynamik durch das Bestehen einer nächtlichen Atmungsstörung weiter verschlechtert wird. Weiterhin könnte die periodische Atmung zur Entwicklung einer Tagesmüdigkeit bei IPAH beitragen, die bekanntermaßen ein häufiges Symptom bei diesen Patienten darstellt [39].

\section{Zusammenfassung}

Die OSA führt im Schlaf zu repetitiven, Apnoe-synchronen Anstiegen des PA-Druckes. Am Tage ist bei 20-30\% der OSA-Patienten eine pulmonale Hypertonie nachweisbar, deren Ausprägungsgrad meistens nur gering ist. Ein wesentlicher pathogenetischer Faktor für die Entstehung der CSR bei Linksherzinsuffizienz ist das Vorliegen einer pulmonalvenösen Hypertonie. Das Krankheitsbild der IPAH kann zu einer nächtlichen periodischen

Druckerhöhung im pulmonalen Gefaßßbett
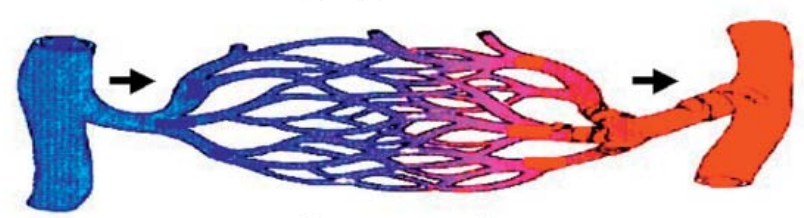

Arterien
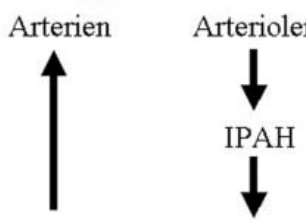

OSA

nächtliche periodische Atmung

Kapillaren

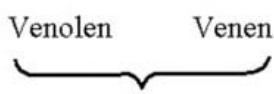

Linksherzinsuffizienz

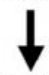

Cheyne-Stokes-Atmung

Abb. 3 Beziehungen zwischen pulmonaler Hypertonie und schlafbezogenen Atmungsstörungen.
Atmung führen, die der CSR sehr ähnlich sieht. Die verschiedenen Beziehungen zwischen pulmonaler Hypertonie und schlafbezogenen Atmungsstörungen sind in Abb. 3 noch einmal graphisch dargestellt.

\section{Literatur}

${ }^{1}$ Atwood CW, McCrory D, Garcia JGN et al. Pulmonary hypertension and sleep-disordered breathing (ACCP evidence-based clinical practice guidelines). Chest 2004; 126: 72S-77S

2 Steiner St, Perings Ch. Pulmonalarterielle Hypertonie und Cor pulmonale bei obstruktiver Schlaf-Apnoe - Pathophysiologie und klinische Relevanz. Internist 1999; 40: 739-746

${ }^{3}$ Steiner St, Strauer BE. Funktionelle Dynamik des rechten Ventrikels und des Lungenkreislaufes bei obstruktiver Schlaf-Apnoe - Therapeutische Konsequenzen. Internist 2004; 45: 1101 - 1107

${ }^{4}$ Marrone O, Bonsignore MR, Romano S et al. Slow and fast changes in transmural pulmonary artery pressure in obstructive sleep apnoea. Eur Respir J 1994; 7: $2192-2198$

${ }^{5}$ Schäfer H, Hasper E, Ewig S et al. Pulmonary haemodynamics in obstructive sleep apnoea: time course and associated factors. Eur Respir J 1998; 12: 679-684

${ }^{6}$ Podszus T, Bauer W, Mayer J et al. Sleep apnea and pulmonary hypertension. Klin Wochenschr 1986; 64: 131 -134

${ }^{7}$ Weitzenblum E, Krieger J, Apprill M et al. Daytime pulmonary hypertension in patients with obstructive sleep apnea syndrome. Am Rev Respir Dis 1988; 138: 345 - 349

${ }^{8}$ Krieger J, Sforza E, Apprill M et al. Pulmonary hypertension, hypoxemia, and hypercapnia in obstructive sleep apnea patients. Chest 1989; 96: $729-737$

${ }^{9}$ Laks L, Lehrhaft B, Grunstein RR et al. Pulmonary hypertension in obstructive sleep apnoea. Eur Respir J 1995; 8: 537- 741

${ }^{10}$ Chaouat A, Weitzenblum E, Krieger J et al. Pulmonary hemodynamics in the obstructive sleep apnea syndrome. Chest 1996; 109: 380-386

${ }^{11}$ Sanner BM, Doberauer C, Konermann M et al. Pulmonary hypertension in patients with obstructive sleep apnea syndrome. Arch Intern Med 1997; 157: $2483-2487$

${ }^{12}$ Sajkov D, Wang T, Saunders NA et al. Daytime pulmonary hemodynamics in patients with obstructive sleep apnea without lung disease. Am J Respir Crit Care Med 1999; 159: 1518 - 1526

${ }^{13}$ Niijima M, Kimura H, Edo $\mathrm{H}$ et al. Manifestation of pulmonary hypertension during REM sleep in obstructive sleep apnea syndrome. Am J Respir Crit Care Med 1999; 159: 1766-1772 
${ }^{14}$ Bady E, Achkar A, Pascal S et al. Pulmonary arterial hypertension in patients with sleep apnoea syndrome. Thorax 2000; 55: 934-939

${ }^{15}$ Alchanatis M, Tourkohoriti G, Kakouros S et al. Daytime pulmonary hypertension in patients with obstructive sleep apnea: the effect of continuous positive airway pressure on pulmonary hemodynamics. Respiration 2001; 68: 566-572

${ }^{16}$ Yamakawa H, Shiomi T, Sasanabe R et al. Pulmonary hypertension in patients with severe obstructive sleep apnea. Psychiatry Clin Neurosci 2002; 56: $311-312$

17 Sajkov D, Wang T, Saunders NA et al. Continuous positive airway pressure treatment improves pulmonary hemodynamics in patients with obstructive sleep apnea. Am J Respir Crit Care Med 2002; 165: $152-158$

${ }^{18}$ McGuire M, Bradford A. Chronic intermittent hypercapnic hypoxia increases pulmonary arterial pressure and haematocrit in rats. Eur Respir J 2001; 18: 279-285

${ }^{19}$ Fagan KA. Physiological consequences of intermittent hypoxia: pulmonary hypertension in mice following intermittent hypoxia. J Appl Physiol 2001; 90: 1600-1605

${ }^{20}$ Javaheri S, Parker TJ, Wexler L et al. Occult sleep-disordered breathing in stable congestive heart failure. Ann Intern Med 1995; 122 487-492

${ }^{21}$ Javaheri S, Parker TJ, Liming JD et al. Sleep apnea in 81 ambulatory male patients with stable heart failure. Types and their prevalences, consequences and presentations. Circulation 1998; 97: 2154-2159

22 Pryor WW. Cheyne-Stokes respiration in patients with cardiac enlargement and prolonged circulation time. Circulation 1951; 14: $233-238$

${ }^{23}$ Naughton M, Benard D, Tam A et al. Role of hyperventilation in the pathogenesis of central sleep apnea in patients with congestive heart failure. Am Rev Respir Dis 1993; 148: 330 - 338

${ }^{24}$ Hanly P, Zuberi N, Gray R. Pathogenesis of Cheyne-Stokes respiration in patients with congestive heart failure: relationship to arterial $\mathrm{PCO}_{2}$. Chest 1993; 104: 1079-1084

25 Traversi E, Callegari G, Bozzoli M et al. Sleep disorders and breathing alterations in patients with chronic heart failure. G Ital Cardiol 1997; 27: $423-429$

${ }^{26}$ Fanfulla F, Mortara A, Maestri R et al. The development of hyperventilation in patients with chronic heart failure and Cheyne-Stokes respiration. Chest 1998; 114: $1083-1090$
${ }^{27}$ Mortara A, Sleight P, Pinna GD et al. Association between hemodynamic impairment and Cheyne-Stokes respiration and periodic breathing in chronic stable congestive heart failure secondary to ischemic or idiopathic dilated cardiomyopathy. Am J Cardiol 1999; 84: $900-904$

${ }^{28}$ Solin P, Bergin P, Richardson M et al. Influence of pulmonary capillary wedge pressure on central apnea in heart failure. Circulation 1999; 99: $1574-1579$

${ }^{29}$ El-Solh AA, Bozkanat E, Mador J et al. Association between plasma endothelin-1 levels and Cheyne-Stokes respiration in patients with congestive heart failure. Chest 2002; 121: $1928-1934$

${ }^{30}$ Lorenzi-Filho G, Azevedo ER, Parker JD et al. Relationship of carbon dioxide tension in arterial blood to pulmonary wedge pressure in heart failure. Eur Respir J 2002; 19: 37-40

${ }^{31}$ Christ M, Grimm W, Rostig S et al. Association of right ventricular dysfunction and Cheyne-Stokes respiration in patients with chronic heart failure. J Sleep Res 2003; 12: 161 - 167

32 Olschewski H, Seeger W. Pulmonale Hypertonie. Bremen: UNI-MED Verlag, 2000

${ }^{33}$ Rafanan AL, Golish JA, Dinner DS et al. Nocturnal hypoxemia is common in primary pulmonary hypertension. Chest 2001; 120: $894-899$

34 Schulz R, Baseler G, Ghofrani HA et al. Nocturnal periodic breathing in primary pulmonary hypertension. Eur Respir J 2002; 19: 658 - 663

${ }^{35}$ Schulz R, Fegbeutel C, Olschewski H et al. Reversal of nocturnal periodic breathing in primary pulmonary hypertension after lung transplantation. Chest 2004; 125: $344-347$

${ }^{36}$ Hanly P, Milar TW, Steljes DG et al. The effect of oxygen on respiration and sleep in patients with congestive heart failure. Ann Intern Med 1989; 111: 777-782

37 Andreas S, Clemens C, Sandholzer H et al. Improvement of exercise capacity with treatment of Cheyne-Stokes respiration in patients with congestive heart failure. J Am Coll Cardiol 1996; 27: 1486 - 1490

${ }^{38}$ Braver HM, Brandes WC, Kubiet MA et al. Effect of cardiac transplantation on Cheyne-Stokes respiration occurring during sleep. Am J Cardiol 1995; 76: 632-634

${ }^{39}$ Rich S, Dantzker DR, Ayres SM et al. Primary pulmonary hypertension. A national prospective study. Ann Intern Med 1987; 107: 216-223 